\title{
Diseño colaborativo aplicado al desarrollo conceptual de embalaje para memorias de resultado de urnas electrónicas
}

Collaborative design applied to conceptual package design for

electronic voting machines' flash drives

\section{Raimundo Lopes Diniz}

Doutor - PPDGg (UFMA); Becario de Productividad en investigación (FAPEMA)

Núcleo de Ergonomía en Procesos y Productos (NEPP)

rl.diniz@ufma.br

$\mathbf{X}$

Rubenio dos Santos Barros

Universidad Federal de Santa Catarina

rubeniobarros@hotmail.com

Zita Carolina González Guzmán

Universidad Federal do Maranhão

zitacgg@gmail.com

PROJËTICA

\section{COMO CITAR ESTE ARTIGO:}

DINIZ, Raimundo Lopes; BARROS, Rubenio dos Santos; GUZMÁN, Zita Carolina González. Diseño colaborativo aplicado al desarrollo conceptual de embalaje para memorias de resultado de urnas electrónicas. Projética, Londrina, v. 12, n.

2, p. 134-159, 2021.

DOI: 10.5433/2236-2207.2021v12n2p134

Submissão: 23-06-2020

Aceite: 20-01-2021 
RESUMEN: El presente artículo describe el desarrollo del rediseño del embalaje para memorias USB de urna electrónica electoral, teniendo como foco central el Diseño Colaborativo (CO- Diseño), presentando resultados relativos a la etapa conceptual. Para el desarrollo del proyecto, se utilizó la Guía para el Desarrollo de Embalajes (GODE) alineándose al concepto de Co-Diseño dentro de un proceso colaborativo, considerando la participación de actores integrados al alcance de los objetivos del proyecto. Los resultados apuntan a la eficiencia del enfoque colaborativo, culminando en un producto de gran aceptación entre los usuarios.

Palabras claves: Embalaje. Rediseño. Pendrives de urna electrónica electoral. Diseño colaborativo.

ABSTRACT: This paper describes the redesign process of the package for flash drives related to the electronic voting devices in Brazil, focusing on the Collaborative Design (Co-Design), up to the conceptual stage. For the development of the project, we used the Packaging Development Guide (GODE) adapted with techniques related to the collaborative design. The results point to the efficiency of the methodological approach, presenting a product of great acceptance among users.

Keywords: Package. Redesign. Electronic voting devices' flash drives. Collaborative design.

\section{INTRODUCCIÓN}

De acuerdo con la Asociación Brasileña de Embalaje (ASSOCIAÇÃO BRASILEIRA DE EMBALAGEM, 2012), los embalajes presentan como funciones básicas: contener, proteger (evitar daños al propio embalaje y al producto contenido) con la viabilidad del transporte de productos y la evolución de las actividades se ha incorporado 
Projética, Londrina, v. 12, n. 2, p. 134-159, agosto 2021

la función de comunicación, y durante la realización de estas funciones, los embalajes son expuestos a diversos riesgos como choques, aceleración, cambios de temperatura, vibración, compresión, oxidación, perforación, aplastamiento, entre otros (ASSOCIAÇÃO BRASILEIRA DE EMBALAGEM, 2012; PEDELHES, 2005).

Dentro del ámbito multidisciplinario, orientado al diseño de embalajes, Fontana, Heemann y Ferreira (2012) afirman que el concepto de productos puede demandar la ampliación de competencias entre el diseñador y otros actores involucrados en el proceso, optimizando así las interacciones en tal concepto y la complejidad del proyecto. Esta ampliación puede ocurrir en forma colaborativa. Heemann, Lima y Scuissiatto (2008), resaltan que el proceso colaborativo en el desarrollo de productos puede aumentar la relación de los diseñadores con otros profesionales. Los autores describen, además, que debido al reconocimiento del diseño como un proceso de trabajo, es posible caracterizarlo como un compromiso compartido para que pueda ser realizado. De esta manera, la actuación del diseñador de forma colaborativa puede ser considerada conveniente y saludable para mejorar las relaciones con otros profesionales señalados en el proceso referido, implementando el intercambio de informaciones y acciones adecuadas para el desarrollo de nuevos productos.

Así, se puede caracterizar el diseño de embalajes, como apto para aplicarse en el proceso colaborativo. Tal hecho pudo ser observado por Negrão y Camargo (2008), quienes describen que el diseño de embalaje además de complejo, envuelve diversas áreas del conocimiento humano: tales como la ingeniería, física, química, psicología, sociología, antropología, diseño, comunicación, producción, marketing, en fin, una gama de conocimientos que no permite un enfoque simple, ya que el embalaje es un sistema multidisciplinario donde la naturaleza de las alteraciones propuestas en un embalaje puede ser distinta por el diseño, material, identidad visual y aún en la mejora de la protección mecánica, fisicoquímica o microbiológica 
del producto, conveniencia en el uso, transporte o legislación. Es decir, una serie de conocimientos que requieren interactuar de manera integrada.

Considerando tales circunstancias, el presente artículo pretende exponer la importancia sobre el uso del enfoque de diseño colaborativo al desarrollo de embalajes, teniendo como caso de estudio el embalaje para memorias de resultado [MR] de las urnas electrónicas. De acuerdo con la BRASIL (2012), las MR son accesorios que tienen como tarea cargar las aplicaciones durante el proceso de iniciación de las urnas, siendo su embalaje enmarcado en la clasificación denominada por la ASSOCIAÇÃO BRASILEIRA DE EMBALAGEM (2012) como embalaje primario, teniendo contacto directo con el producto y que atiende a los aspectos pragmáticos del proyecto (el de contener), debiendo proteger y conservar la integridad química y física del producto, minimizando la absorción de gases (oxígeno, gas carbónico) humedad, olores, luz, etc. Las MR exigen un embalaje que sea seguro y adecuado a las necesidades de protección y acondicionamiento de estos dispositivos, pues son elementos de gran relevancia, en el funcionamiento de las urnas electrónicas, y por lo tanto contribuyen directamente en el proceso electoral brasileño.

\section{LA URNA ELECTRÓNICA Y LAS MR}

Antes de que se pueda detallar las configuraciones de las MR, es necesario describir el conjunto del cual forma parte la urna electrónica. Según la definición del Tribunal Superior Electoral (BRASIL, 2010), la Urna Electrónica - UE (figura 1) es un microcomputador de uso específico para elecciones y se presenta con recursos de seguridad, como el barajado interno de los datos, que garantiza el derecho secreto del voto y la confiabilidad del proceso de votación. Este equipamiento genuinamente brasileño, además de seguridad, agrega facilidad, agilidad y transparencia al proceso electoral, eliminando el riesgo de fraude. 
Projética, Londrina, v. 12, n. 2, p. 134-159, agosto 2021

Figura 1 - Modelo de urna electrónica.

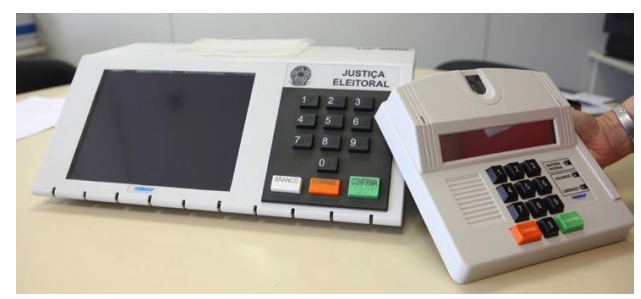

Fuente: Tribunal Superior Eleitoral (2010).

Todos los modelos de urnas electrónicas existentes están compuestos por dos módulos principales: terminal del elector y el micro terminal, este último también llamado terminal del mesero. La terminal del elector es el módulo donde se hace la interacción entre la urna y el elector, registrando numéricamente el voto. La terminal del mesero tiene la función de habilitar a los electores para votar y de realizar el seguimiento del proceso de votación (BRASIL, 2010, 2012)

La urna electrónica está compuesta por otros equipamientos periféricos, mecanismos internos y accesorios que garantizan su funcionamiento; entre estos equipamientos periféricos se encuentra la Memoria de Resultado. (M.R.) De acuerdo con la Tribunal Superior Eleitoral (BRASIL, 2012), las memorias de resultado (figura 2) son dispositivos de almacenamiento de datos Memorias USB de uso exclusivo en las urnas, que contienen información necesaria y complementaria para el funcionamiento del sistema, siendo su principal función el almacenamiento del resultado de las mismas votaciones para su posterior transmisión y recuento.

Figura 2 - Modelos de MR.

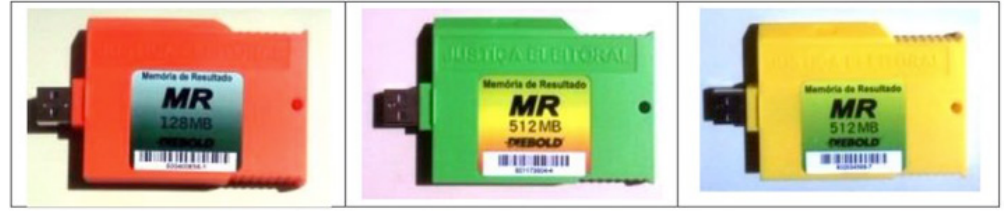

Fuente: Brasil (2012). 
En el proceso de iniciación de la urna, ella realiza la lectura de la MR y carga las aplicaciones correspondientes previamente transferidas (BRASIL, 2012). Las memorias de resultado también desempeñan un papel fundamental en la resolución de algunos problemas que puedan surgir durante el proceso de votación (fecha y hora incorrectas, fallo de periféricos, etc.) ya que, de acuerdo con el Manual de Ejecución de Urnas Electrónicas (BRASIL, 2012), en algunas situaciones, si hay defecto en la comunicación entre la MR y la urna, es necesario la sustitución de la urna electrónica, lo que resalta su importancia. Incluso durante el proceso de sustitución de urnas defectuosas por nuevas (urnas de contingencia) las MR son elementos clave, ya que son responsables de transmitir los datos que permitan esa sustitución. Es así que las memorias de resultado (MR) son esenciales para el funcionamiento de las urnas electrónicas (BRASIL, 2012).

Las MR también se relacionan con una serie de conexiones entre sectores que se generan a partir de las funciones desempeñadas por estos medios, donde la preservación de la integridad de las MR durante el acondicionamiento es de sustancial contribución al adecuado funcionamiento de las votaciones y para evitar resultados desproporcionados entre los procesos y sectores involucrados (BRASIL, 2012). Estas premisas también son importantes en el desarrollo de envases dirigidos al soporte del proceso electoral brasileño, donde los embalajes desempeñan un papel importante en el acondicionamiento de la urna electrónica y sus componentes necesarios, que incluyen las MR, para la realización de las elecciones.

El alto nivel de confiabilidad esperado de los procedimientos del Proceso Electoral, que engloban las actividades de manipulación, transporte, operaciones de software y acondicionamiento de las MR, resalta la importancia de un sistema de embalaje apropiado para esos medios que tengan en cuenta la función práctica (de uso), el enfoque a los aspectos funcionales para optimizar las actividades ejecutadas y que esté alineado a las características y limitaciones de los factores humanos involucrados. 
Projética, Londrina, v. 12, n. 2, p. 134-159, agosto 2021

El alto nivel de confiabilidad esperado de los procedimientos del Proceso Electoral, que engloban las actividades de manipulación, transporte, operaciones de software y acondicionamiento de las MR, resalta la importancia de un sistema de embalaje apropiado para esos medios que tengan en cuenta la función práctica (de uso), el enfoque a los aspectos funcionales para optimizar las actividades ejecutadas y que esté alineado a las características y limitaciones de los factores humanos involucrados.

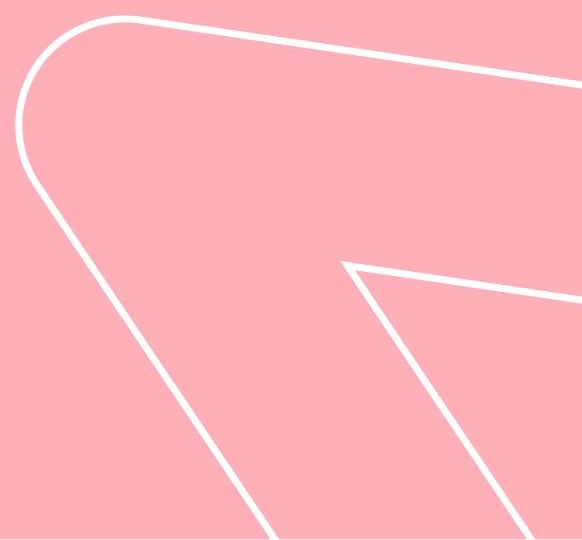




\section{LA RELEVANCIA CIENTÍFICA DE LA COLABORACIÓN EN EL DISEÑO}

De acuerdo con Heemann, Lima y Scuissiatto (2008), la colaboración en el proceso de diseño se presenta como una relevancia científica. Para los autores, tal relevancia es pautada en la presión que hay bajo los diseñadores en cuanto a la atención a múltiples requisitos proyectivos, en un corto espacio de tiempo, demandando así formas mejoradas de trabajo para la concepción de nuevos productos.

Para Penha et al. (2010), el 'Diseño Colaborativo' debe considerar un enfoque más humano, siendo transformador de un mero rediseño mecánico, resultante del proceso de diseño (aplicación metodológica), a un rediseño resultante de la naturaleza de las interacciones de los actores involucrados en el proceso, aun pudiendo afectar las tomas de decisiones en cuanto al desarrollo del proceso del proyecto. Segundo Fontana, Heemann y Ferreira (2012), el diseño colaborativo ha ocurrido, de forma frecuente, en estudios e investigaciones del área por el hecho de que las actividades proyectivas se presentan, cada vez más, complejas. Esta complejidad tiene relación con nuevas tecnologías y sistemas provenientes de las demandas del mercado contemporáneo, el cual viene exigiendo la actuación de equipos multidisciplinarios para la ejecución de diversas tareas específicas que deben congregar a los objetivos del proyecto de manera integrada.

Hay varios conceptos acerca de la colaboración en el diseño. En el presente artículo, se utilizará como referencia principal el concepto descrito por Fontana (2012, p. 37): “(...) Es un esfuerzo recíproco entre personas de iguales o diferentes áreas de conocimiento, separadas físicamente o no, con el objetivo común de encontrar soluciones que pueden satisfacer a todos los interesados". Esto puede suceder compartiendo información y responsabilidades, organizando tareas y recursos, administrando múltiples perspectivas y creando un entendimiento compartido en un proceso de diseño. La colaboración busca producir un producto y/o servicio consistente y completo a través de una gran variedad de fuentes de información 
Projética, Londrina, v. 12, n. 2, p. 134-159, agosto 2021

con cierto grado de coordinación de las diversas actividades implementadas. Este proceso depende de la relación entre los actores involucrados, la confianza entre ellos y la dedicación de cada parte.

La parte de la planificación estratégica en cuanto a la operación, ejecución de las acciones humanas frente a las tareas prescritas, puede definir el alcance de la colaboración. De manera general, tal planificación enfoca el problema a ser resuelto trazándose, así, una táctica operacional, es decir: "lo que debe ser hecho". Tal táctica puede ser considerada, en principio, como teórica a partir del delineamiento de metas a ser alcanzadas, o sea: "como debe ser hecho". Operacionalmente, se tiene la práctica, la ejecución planificada de donde se quiere llegar para la resolución del problema. En un sesgo organizacional, hay una organización jerárquica y coherente entre la táctica operacional y su aplicabilidad, siendo coherente que ocurra lo siguiente: "la operación sea jerárquicamente englobada por la táctica, que a su vez sea englobada por la estrategia". Tal estructura jerárquica puede ocurrir en la operación compartida, siendo denominada "la Colaboración" (HEEMANN; LIMA; SCUISSIATTO, 2008).

Para Fontana (2012), la inserción de la premisa "Colaboración" en el desarrollo proyectual o en el proceso de diseño, puede apuntar a múltiples criterios, caracterizando las acciones en un ámbito interdisciplinario. Así, en este contexto, existe la elevación de la complejidad del proceso, ampliando la red entre los actores involucrados en las operaciones proyectuales.

Por lo tanto, durante el proceso del diseño de embalaje, el diseñador o equipo de diseño, puede beneficiarse de la aproximación y colaboración de diversos actores en los más diversos ámbitos, desde las fases iniciales, donde pueden ser recogidas y filtradas informaciones de delineamiento y comprensión de la demanda (problematización), hasta las etapas finales, como la elección de propuestas de solución, teniendo una visión más precisa y acorde con la realidad 
a partir de quién realmente utilizará el producto final. Se percibe entonces que la colaboración puede propiciar un enfoque de cambio no sólo enfocado únicamente en el embalaje, sino en el proceso en que está inserta.

\section{MÉTODOS Y TÉCNICAS}

Se puede caracterizar la presente investigación como descriptiva, con aplicabilidad a un estudio de caso. La investigación descriptiva busca observar fenómenos científicos generando la descripción, clasificación e interpretación por el conocimiento de la realidad, sin interferirla. En el estudio de caso, se encuentra la realización de la investigación en un objeto particular de estudio (RUDIO, 1997). De esta forma, se pretende abordar el fenómeno del acondicionamiento de MR, enfocándose en el reajuste del embalaje, considerando la aplicación del enfoque 'Diseño Colaborativo'.

Para la aplicación del diseño colaborativo, se utilizó la clasificación de Lima y Heemann (2009), los cuales clasifican la colaboración proponiendo "líneas colaborativas" que tratan de las relaciones entre los miembros (participantes) involucrados con el proceso y que influencian directamente en el resultado final del proyecto. Lima y Heemann (2009) destacan que tales relaciones presentan características inherentes de cada línea colaborativa y su importancia en el resultado final. En el caso del presente trabajo, se usó la clasificación que evidencia la colaboración entre algunos miembros pertenecientes a un proyecto, por motivo de división de tareas (figura 3).

Figura 3 - Relación entre algunos miembros.

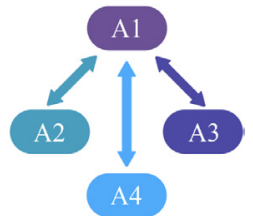

Fuente: adaptado de Lima e Heemann (2009). 
Projética, Londrina, v. 12, n. 2, p. 134-159, agosto 2021

Con relación al desarrollo del embalaje, se utilizó el método GODE - Guía de Orientación para el Desarrollo de Embalajes (MERINO, 2002). El método GODE se recomienda específicamente al diseño de embalajes, teniendo en cuenta criterios y elementos que son intrínsecos a este tipo de producto. Los pasos del GODE se describen como:

A) Etapa 0 - pedido: describe el detalle inicial de los problemas en el embalaje, además de información acerca de los usuarios y de las cuestiones de uso. Esta etapa fue realizada por medio de visitas preliminares en campo, observaciones sistemáticas y asistenciales, además, entrevistas no directivas y focalizadas acerca del contexto funcional de las MR, considerando el abordaje de Moraes y Mont'Alvão (2009).

B) Etapa 1 - búsqueda de los datos: estudio de campo, con una investigación del producto a fin de conocer los medios de producción, materiales empleados y embalajes de propósito equivalente o similar. De esta forma, se levantaron y se analizaron 40 modelos de embalajes similares (acondicionamiento de medios de almacenamiento de datos) $y$, a continuación, sus configuraciones fueron estudiadas de acuerdo a la clasificación de los tipos de embalaje según la ABRE y según los análisis de producto propuestos por Löbach (2001). La idea sería anticipar imprevistos y situaciones de riesgo en cuanto al desarrollo de la estrategia de diseño (BAXTER, 1998; MESTRINER, 2002).

C) Etapa 2 - análisis, interpretación y organización de datos: asignación de las características del embalaje actual, realizando el análisis estructural, funcional, de los materiales y de los procesos de fabricación, y de la configuración que fueron descritos por Löbach (2001). A continuación, se presentan los requisitos proyectivos utilizando la categorización de Rodríguez (1986).

D) Etapa 3 - fase de creación: generación de alternativas de diseño, sobre la base de la definición del concepto que se estableció para el embalaje. 
En esta etapa, se utilizan técnicas que estimulan el pensamiento creativo, siendo ellas el Brainstorming, Brainwritting y el Círculo de Oportunidades. Las ideas fueron evaluadas en el contexto colaborativo, entre el equipo de diseño y los usuarios potenciales, además de profesionales especialistas. Las herramientas adoptadas en ese sentido fueron las técnicas de evaluación y recolección de opinión, como la técnica ThinkingOutLoud, y la Evaluación Cooperativa entre usuarios y especialistas (BAXTER, 1998; MORAES; SANTA ROSA, 2012).

Además, para tener una visión más amplia acerca de ciertos aspectos técnicos, se utilizó la Técnica Delphi (MORAES; SANTA ROSA, 2012), que consiste en la recolección de opiniones con especialistas. Su aplicación consistió en la presentación del proyecto, su desarrollo y la problemática para especialistas del área del diseño, de materiales y procesos, estética y de la ergonomía, a fin de que profesionales nortean el progreso del proyecto de acuerdo con la tecnicidad y el conocimiento específico de cada área que se desempeñan (BAXTER, 1998; MORAES; SANTA ROSA, 2012;).

Para el proceso de creación, discusión y preselección de las ideas que se mostraron prometedoras, y que podrían ser desarrolladas más a fondo, se elaboraron representaciones a nivel de maqueta electrónica a través de softwares de creación y edición de imagen (Adobe Illustrator ${ }^{\circledR}$ y Photoshop ${ }^{\circledR}$ versiones Creative Suite 6 de 2012 y Creative Cloud 2015.0.0, Google Sketchup Pro® versión 16.0.19913, Autodesk AutoCAD® versiones 2012 y 2014), así como el programa de rendering LuxionKeyshot versiones 2015 y 2016. En conjunto con el desarrollo de ideas, fueron elaborados cuadros comparativos entre posibles procesos de producción y materiales, haciendo desde ya una selección preliminar basada en la literatura (LIMA, 2006; NEGRÃO; CAMARGO, 2009) para ser presentados en seguida a los especialistas y usuarios. 
Projética, Londrina, v. 12, n. 2, p. 134-159, agosto 2021

E) Etapa 4 - ejecutiva: en esta fase, el equipo de diseño planeará la configuración y validación de la idea elegida (MERINO; CARVALHO; MERINO, 2007), además de su producción.

Se hace hincapié en que en el presente artículo sólo se presentarán los resultados relativos al desarrollo de propuestas conceptuales en cuanto al reajuste del embalaje antes citado.

\section{RESULTADOS Y DISCUSIONES}

\subsection{Paso 0: Pedido del trabajo}

A través de las visitas, observaciones y entrevistas, se percibió que la demanda de acondicionamiento de MR en el Tribunal Regional Electoral [TRE-MA] analizado corresponde a un total de cerca de 42 mil MR que auxilian en el funcionamiento de 20.202 urnas electrónicas. Su acondicionamiento se hace dentro de cajones plásticos que a su vez se quedan en armarios dentro del Foro del Tribunal.

Cuando hay necesidad de movimiento de las MR, esa demanda puede ser suplida de formas diversas, siendo realizado desde el transporte de unidades manualmente, hasta el uso de cajas de cartón para auxiliar en el transporte de grandes cantidades. Pero el medio más utilizado es con el auxilio de las maletas plásticas, que son importantes al transporte de las MR a regiones en el interior de todo el estado al que se destina el Tribunal.

El embalaje de las MR de urnas electrónicas se enmarca en la clasificación denominada por la ASSOCIAÇÃO BRASILEIRA DE EMBALAGEM (2012) como embalaje primario, que es aquel que está en contacto directo con el producto y que atiende a los aspectos pragmáticos del proyecto (contener). Cada maleta puede acomodar 50 MR, siendo que hay un total de 744 maletas plásticas, lo que posibilita el transporte de hasta $37.200 \mathrm{MR}$. 
Diseño colaborativo aplicado... electrónicas

DINIZ, R. L.; BARROS, R. S.; GUSMÁN, Z. C. G.

\subsection{Paso 1: Recopilación de datos}

Entre las actividades involucrando $\mathrm{MR}$, las que exigen el mayor volumen de movimiento de MR son el envío de las MR a las Zonas Electorales (locales de votación) durante el período de elección, el mantenimiento preventivo y el mantenimiento correctivo, donde todas se utilizan en algún momento de las maletas plásticas.

En los modelos de embalaje levantados, en el $67 \%$ de los casos (27), sólo se encontraron embalajes primarios. En los otros 13 modelos (33\%) había la presencia tanto de embalajes primarios como secundarios. La ASSOCIAÇÃO BRASILEIRA DE EMBALAGEM (2012) considera como embalaje primario el que tiene contacto directo con el producto envasado y lo secundario siendo el que contiene uno o más envases primarios.

Los formatos entre los envases encontrados varían: bandeja (60\%), estuche (27\%) y maletín (13\%), el modelo con mayor capacidad fue la bandeja, comportando 120 medios, destinada a tarjetas de memoria. La gran mayoría de los modelos (70\%), utilizó sólo un material en su composición, siendo los más utilizados el Poliestireno - PS y el Politereftalato de Etileno - PET, que son commodities.

Como un panorama general construido a partir de los análisis propuestos por Löbach (2001), se puede afirmar que, sobre la posibilidad de apilamiento, sólo 4 (10\%) modelos no presentaron esa función. Para la apertura y cierre de los envases, todas las maletas presentaban algún sistema de cierre o lacre, mientras que esa función aparecía en el 90,9\% de los estuches y apenas entre el 8,3\% de las bandejas. En cuanto a los colores, el $45 \%$ tenía sólo un color, el 30\% eran transparentes / incoloros, el 17\% tenía dos colores y el $8 \%$ tenía 3 o más colores, siendo el negro el color más utilizado.

De esta forma se generó el actual panorama existente en el mercado para el acondicionamiento de medios de almacenamiento, presentando una gama de 
Projética, Londrina, v. 12, n. 2, p. 134-159, agosto 2021

configuraciones que posteriormente fueron filtradas y ayudó en el proceso de generación de ideas.

\subsection{Paso 2: análisis, interpretación y organización de datos}

Se constató que el embalaje existente de las MR presenta problemas estructurales que acaban por interferir en el modo de usoy en sus funciones. No hubo preocupación en relación al análisis de la resistencia del material y de su dimensionamiento, lo que acarrea en un exceso de flexibilidad, pudiendo comprometer la organización y retención de las MR, además de interferir en el apilamiento, manipulación y vida útil de los embalajes (figura 4).

Figura 4 - Problemas detectados en el embalaje.
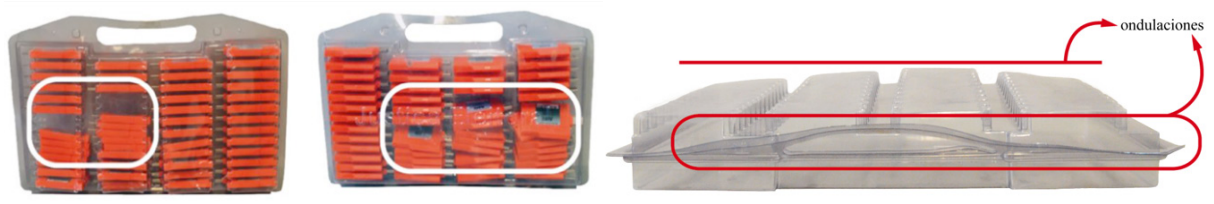

Fuente: achivo proprio del autor

Se observó también que el embalaje presenta fragilidades en su estructura resultantes del proceso de producción y del material escogidos (fabricación por termoformado con uso del polímero Politereftalato de Etileno - PET) que pueden comprometer su cuerpo como consecuencia del uso, acarreando ranuras, trincas, ondulaciones y áreas amasadas o quebradas.

Se observó que el punto de plegado no presentaba una resistencia adecuada, presentándose roto en algunas áreas, resultando en "cantos vivos" y 
áreas puntiagudas, lo que muestra una fuente de incomodidad y de riesgo para el individuo. El subsistema de cierre no fue considerado eficaz por no garantizar el total cierre y al mismo tiempo de difícil apertura, haciendo que sea destinada más fuerza que el ideal para que la tapa sea abierta. El área de empuñadura se presentó sin conformación con los requisitos de forma y dimensionamiento antropométrico, además de presentar rebabas y bordes cortantes resultantes del mal acabado superficial y del proceso de producción. Estas configuraciones van en contra de las recomendaciones ergonómicas presentadas por Lida y Guimarães (2016), que afirman que la presencia de protuberancias y rebabas en la empuñadura son perjudiciales porque concentran presiones en esos puntos.

Ante la información de esta etapa y de las etapas anteriores, fue posible generar el briefing basado en Rodríguez (1986), en el cual se definió que, para los requisitos de uso, existe la necesidad de tener una mejor contención de las MR dentro del embalaje de modo que la lectura del código de barras (patrimonio) sea fácil y se dé de forma ágil. En lo que se refiere a la seguridad, el embalaje no debe contener riesgos al usuario, eliminando, por lo tanto, cantos vivos, rebabas y bordes cortantes, como sugiere Lida y Guimarães (2016), al tiempo que esa contención no permite movimientos indeseados de MR.

Para la manipulación y antropometría, se consideran las directrices de Tilley (2005) y lida y Guimarães (2016) para controles manuales y manejos, en el cual recomiendan área para empuñaduras de forma cilíndrica entre 3 a $5 \mathrm{~cm}$ (IIDA; GUIMARÃES, 2016) o 32 a 38 mm (TILLEY, 2005), de modo que se obtenga comodidad para el usuario.

Hay una necesidad de adecuación del embalaje en cuanto a la eficiencia, eficacia y satisfacción durante el uso, pudiendo ser citada la necesidad de adecuación en cuanto al proceso de inserción y remoción de las MR, la fuerza demandada y la eficiencia en el accionamiento de la apertura y cierre del embalaje. En cuanto a la 
Projética, Londrina, v. 12, n. 2, p. 134-159, agosto 2021

percepción, es necesario proporcionar la comprensión de acondicionamiento de las MR, así como la forma en que se realiza el transporte, presentando elementos explicativos en cuanto a su función principal y facilidad en la lectura del código de barras de las MR (patrimonio).

Para los requisitos de función, se hace necesario el desarrollo de un sistema de apilamiento. Es válida la inclusión de un sistema de apertura y cierre en los embalajes de una forma en que no haya posibilidad de apertura accidental o demasiado esfuerzo físico de los usuarios. Es necesario permitir el acondicionamiento y apilamiento tanto vertical y horizontal de los envases, evidenciando la facilidad de almacenamiento. Para la resistencia, es crucial el desarrollo de posibilidades que permitan al embalaje soportar esfuerzos en cuanto a compresión, tensión o choque, caídas, agentes externos (agua, humedad, corrosión, intemperies y eventuales animales), fricciones y superposición de pesos, preservando las MR. Sobre los requisitos estructurales, se debe pensar una cantidad reducida de componentes, partes y elementos que constituirán la propuesta del embalaje.

La lista de requisitos pasó por la evaluación colaborativa tanto de los usuarios finales y de los especialistas, principalmente junto al especialista en materiales y procesos y al especialista en ergonomía, habiendo sido aprobado por todos los participantes (figura 5).

Figura 5 - Procesos de evaluación colaborativa.
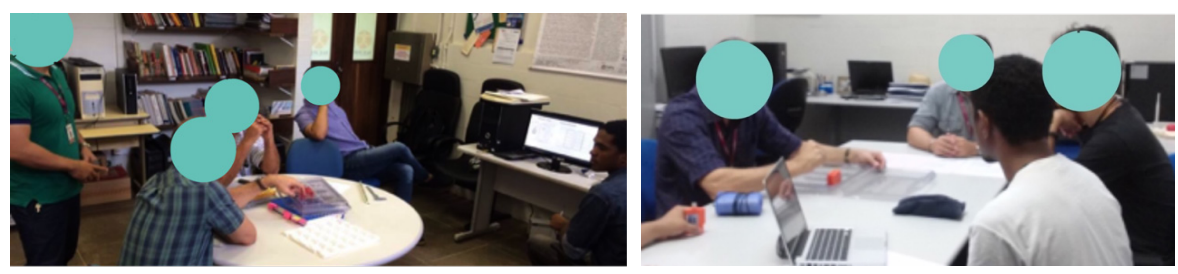

Fuente: achivo proprio del autor 


\subsection{Paso 3: fase de creación}

Antes del proceso creativo, ocurrieron discusiones sobre qué elementos y características serían capaces de proporcionar innovación, y asegurar la practicidad y la funcionalidad de la propuesta del nuevo embalaje. Por ser uno de los puntos de diferenciación, el factor ergonómico ganó especial atención, buscando alternativas, referencias, normas y recomendaciones que garanticen la mejor interacción de los usuarios con el producto. El proceso creativo ocurrió en diversos momentos de esta fase, siendo inicialmente realizado por el equipo de diseñadores (en total de 3), bajo la supervisión del coordinador del proyecto (también profesional del área de diseño). Cómo tenía un carácter libre, surgieron alternativas variadas en cuanto al enfoque de solución de la demanda, como propuestas que presentaban orientaciones diversas de los embalajes y del acondicionamiento de las MR, embalajes de niveles primario y secundario, con diferentes tipos de materiales y accesorios, además de otras especificaciones (figura 6).

Figura 6 - Generación de ideas.

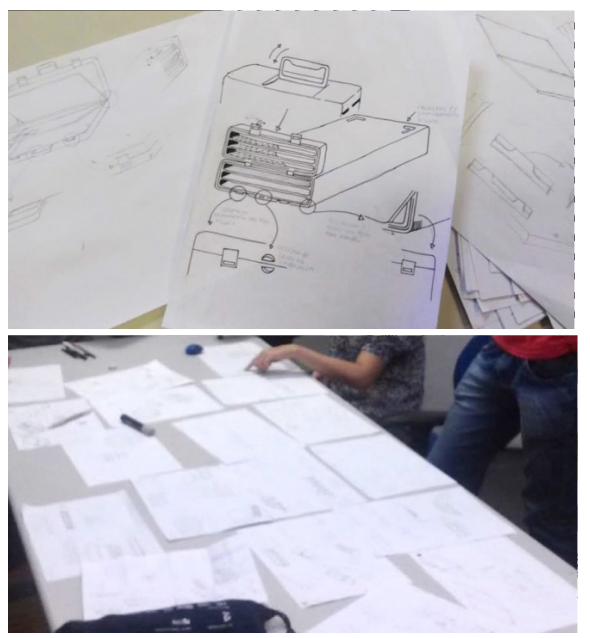

Fuente: achivo proprio del autor 
Projética, Londrina, v. 12, n. 2, p. 134-159, agosto 2021

A continuación, las propuestas generadas (figura 7).

Figura 7 - Principales ideas generadas.
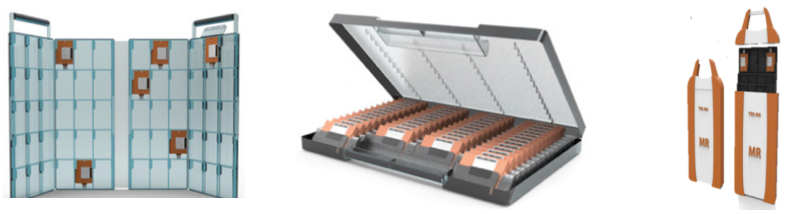

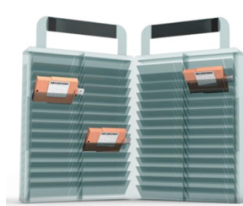

Fuente: achivo proprio del autor

Durante las presentaciones de las alternativas a través de la Evaluación Cooperativa y de la Técnica Delphi, la alternativa que más se destacó tanto entre los usuarios como entre los especialistas fue la propuesta " $D$ ", el cual utilizó mejor el espacio disponible $y$, además, presentó la mejor forma de acondicionamiento de las MR. Hubo la concordancia en la elección tanto de los usuarios como de los especialistas sobre la misma propuesta, siendo destacada la eficiencia en la alineación de pensamiento. Para las alternativas sobre el proceso de producción y materiales, el especialista en materiales y procesos indicó como mejor solución el proceso de inyección y el Polipropileno (PP) como el material más viable. Estando de acuerdo con el briefing propuesto y con las necesidades destacadas por los usuarios.

El proceso colaborativo posibilitó la construcción de un conjunto de mejoras que surgieron a partir o durante la interacción entre los actores involucrados. La Figura 9 desarrollada a partir del modelo propuesto por Lima y Heemann (2009), donde se muestra claramente como cada individuo o grupo de individuos contribuyó durante el desarrollo del proyecto, siendo posible observar sus interacciones de acuerdo con las etapas de desarrollo. El gran número de interacciones refleja la integración de las acciones que llevaron hasta las proposiciones de mejoras. 
Diseño colaborativo aplicado... electrónicas DINIZ, R. L.; BARROS, R. S.; GUSMÁN, Z. C. G.

Se observa, además, que la participación de algunos colaboradores trascendió a determinadas etapas, lo que es el resultado del impacto de las especificidades de determinados colaboradores para alcanzar los requisitos de diseño. Este es el caso de la contribución del especialista en materiales, que tuvo una relación directa con la comprensión del muestreo de similares y en la construcción estructural del embalaje y también de la actuación de los jefes de sección, así como de los técnicos de urnas para proporcionar el punto de vista del usuario a lo largo del proceso de rediseño para que los resultados estuvieran en concordancia con la real demanda.

Figura 9 - Representación del proceso colaborativo.

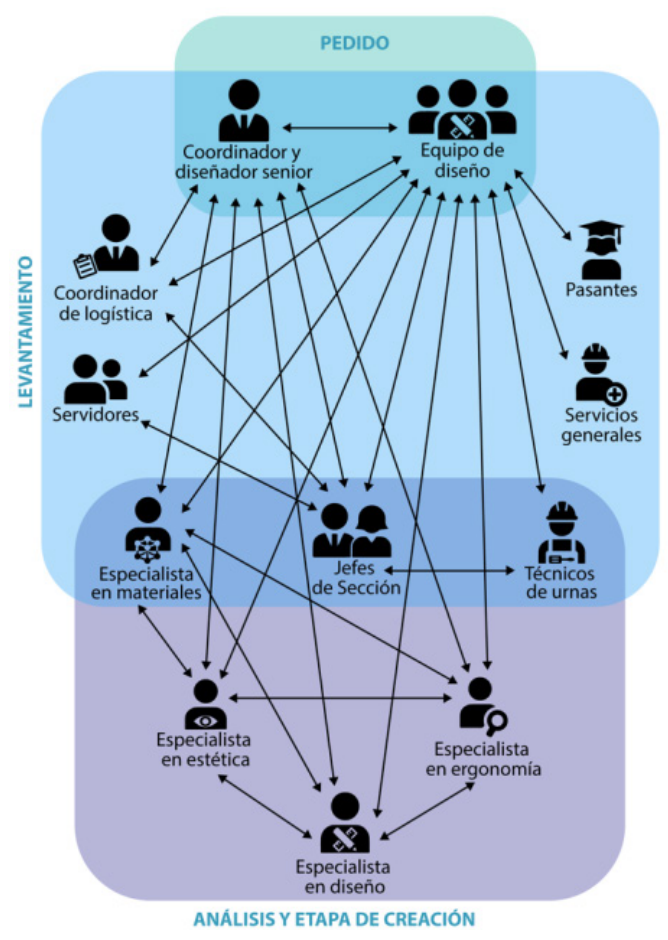

Fuente: achivo proprio del autor 
Projética, Londrina, v. 12, n. 2, p. 134-159, agosto 2021

Resumidamente, los puntos positivos de la nueva maleta pueden ser listados como: mayor facilidad en la lectura del patrimonio; mayor resistencia del embalaje a las fuerzas externas, resultante de una serie de especificaciones del proyecto, como mayor espesor de las paredes del embalaje, estructuras internas de soporte, mejor organización de las MR de modo a evitar espacios ociosos, entre otros; mayor durabilidad del material escogido. El material utilizado garantiza protección electrostática (ESD), pues según Bucci (2010), el plástico promueve tal protección, lo que es importante para componentes sensibles, como las memorias flash (lo que incluye las MR); menor ocupación de espacio y eliminación del área destinada para el apilamiento de las maletas en el área de suministros en el Tribunal; nuevas posibilidades de gestión de las MR, con eliminación de etapas y agilización de procedimientos (facilitación de la lectura de patrimonio); ganancia en comodidad en el transporte y manejo del embalaje; eliminación de apilamiento irregular; posibilidad de apilamiento vertical y horizontal de los embalajes; eliminación de movimientos no deseados de MR dentro del embalaje; sistema de apertura y cierre más eficientes; mayor seguridad para el usuario con la eliminación de factores de riesgo como cantos vivos y rebabas; y más seguridad para el producto acondicionado (MR).

\section{CONSIDERACIONES FINALES}

Es posible afirmar, a partir de los resultados obtenidos, la eficiencia del enfoque colaborativo. A lo largo de todo el proceso, la colaboración fue importante en el proceso de desarrollo del embalaje, contando con la visión de varios actores (como: diseñadores, ergonomistas, especialistas en materiales, en estética y en planificación gráfica, además de los usuarios potenciales - empleados del TRE-MA).

El proceso creativo constante resultó en varios enfoques para la atención de la demanda, abordajes que sufrieron diversas evaluaciones y mejoras pautadas en las herramientas y métodos preseleccionados, así como la constante búsqueda 
A lo largo de todo el proceso, la colaboración fue importante en el proceso de desarrollo del embalaje, contando con la visión de varios actores (como: diseñadores, ergonomistas, especialistas en materiales, en estética y en planificación gráfica, además de los usuarios potenciales - empleados del TRE-MA).(.) el diseño colaborativo para la resolución del problema, potencializó e impactó directamente en el carácter de innovación de la propuesta seleccionada.

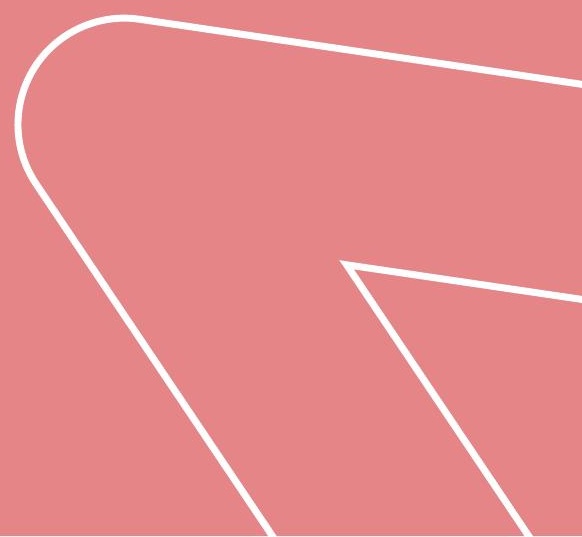


Projética, Londrina, v. 12, n. 2, p. 134-159, agosto 2021

por la mirada técnica de especialistas de diferentes áreas. Este filtro reforzó la calidad de la propuesta final desarrollada.

La propuesta conceptual puede abrir nuevas posibilidades de gestión y almacenamiento de MR, ya que la inserción del embalaje en situación real permitiría ahorrar espacio, mejor organización y la eliminación de cajones plásticos dentro de los armarios, resultando en una reducción a través de la sustracción de ese recipiente, y una mayor comodidad y practicidad durante el uso.

Destaquemos que el diseño colaborativo para la resolución del problema potencializó e impactó directamente en el carácter de innovación de la propuesta seleccionada. Esto fue debido a la diversidad y posibilidad de fuentes de información proporcionadas por el método, garantizando la toma de decisiones de forma más consciente en conjunto. $Y$ aunque la inclusión de más personas en el proceso de diseño puede no ser algo relativamente simple y dar lugar a situaciones no planeadas, es justamente en este factor que reside un punto importante construido a través de ese abordaje. Pues, cuando propiamente dirigidos a un objetivo común, esas personas, situaciones y posibilidades enriquecen el proyecto en su resultado final.

\section{Agradecimientos}

AI TRE, por possibilitar el desarrollo de esta investigación; a la FAPEMA, por el auxilio financiero; al Núcleo de Ergonomía y Productos (NEPP); a Larissa Ferro, Moisaniel Pimentel, Caio Oliveira, Denilson Santos, Francisco Lobo, Lívia Campos, João Raposo, Lucilene Cardoso e Bruno Carvalho 
Diseño colaborativo aplicado al... electrónicas DINIZ, R. L.; BARROS, R. S.; GUSMÁN, Z. C. G.

\section{REFERENCIAS}

1. ASSOCIAÇÃO BRASILEIRA DE EMBALAGEM. 0 papel e funções da embalagem. São Paulo: ABRE, 2012.

2. BAXTER, Mike. Projeto de produto: guia prático para o desenvolvimento de novos produtos. São Paulo: Editora Edgard Blücher, 1998.

3. BRASIL. Tribunal Superior Eleitoral. Manual de exercitação de urnas eletrônicas: modelos: UE2004, UE2006, UE2008, UE2009, UE2010, UE2011. Brasília, DF: TSE, 2012. Disponível em: https://docplayer.com.br/28156626Manual-de-exercitacao-de-urnas-eletronicas.html. Acesso em: 23 abr. 2020.

4. BRASIL. Tribunal Superior Eleitoral. Por dentro da urna. 2. ed. Brasília, DF: TSE, 2010.

5. BUCCl, Doris Zwicker. Processo de desenvolvimento de produtoembalagem: uma proposta orientada à sustentabilidade. 2010. Tese (Doutorado em Engenharia Mecânica) - Universidade Federal de Santa Catarina, Florianópolis, 2010.

6. FONTANA, Isabela Mantovani. Fatores críticos de sucesso para a colaboração no design de sistemas produto-serviço. 2012. Dissertação (Mestrado em Design) - Universidade Federal Paraná, Curitiba, 2012.

7. FONTANA, Isabela Mantovani; HEEMANN, Adriano; FERREIRA, Marcelo Gitirana Gomes. Fatores críticos de sucesso para a colaboração no design de sistemas produto-serviço. Design \& Tecnologia, Porto Alegre, v. 2, n. 4, p. 53-60, dez. 2012.
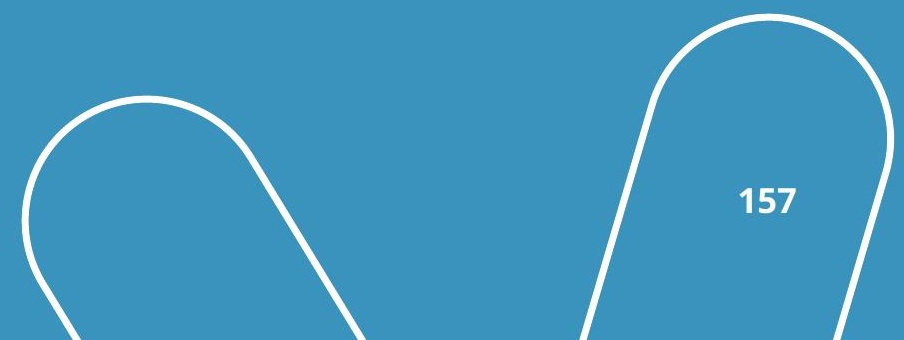
Projética, Londrina, v. 12, n. 2, p.134-159, agosto 2021

8. HEEMANN, Adriano; LIMA, Patrícia Jorge Vieira; SCUISSIATTO, Jeandrey Corrêa. Compreendendo a colaboração em design de produto. In: DISEÑO EN PALERMO, ENCUENTRO LATINOAMERICANO DE DISEÑO, 2008, Palermo. Anais [...], Palermo: Universidad de Palermo, 2008. Disponível em: https://www.researchgate.net/profile/Adriano-Heemann/ publication/267380103_Compreendendo_a_Colaboracao_em_Design_de_ Produto/links/546f760b0cf2d67fc0311500/Compreendendo-a-Colaboracaoem-Design-de-Produto.pdf. Acesso em: 23 abr. 2020.

9. IIDA, Itiro; GUIMARÃES, Lia Buarque de Macedo. Ergonomia: projeto e produção. 3. ed. São Paulo: Blucher, 2016.

10. LIMA, Marco Antonio Magalhães. Introdução aos materiais e processos para designers. Rio de Janeiro: Ciência Moderna, 2006.

11. LIMA, Patricia Jorge Vieira; HEEMANN, Adriano. Premissas para o alcance do trabalho colaborativo em design. In: CONGRESSO INTERNACIONAL DE PESQUISA EM DESIGN, 5., 2009, Bauru. Anais [...]. Bauru: CIPED, 2009. p. 1-7. Disponível em: https://issuu.com/pet_design/docs/premissas-para-oalcance-do-trabalho-colaborativo. Acesso em: 23 abr. 2020.

12. LÖBACH, Bernd. Design industrial: bases para a configuração de produtos industriais. Paulo: Edgard Blücher, 2001.

13. MERINO, Eugenio. Gestão de design: inovação e integração. ABC Design, Curitiba, n. 2, p. 18-20, 2002. 
14. MERINO, Eugenio; CARVALHO, Roberto Luiz; MERINO, Giselle. Design e processo de concepção: guia de orientação para o desenvolvimento de embalagens. Florianópolios: UFSC, 2007.

15. MESTRINER, Fábio. Design de embalagem: curso básico. 2. ed. São Paulo: Prentice Hall, 2002.

16. MORAES, Anamaria de; MONT'ALVÃO, Cláudia. Ergonomia: conceitos e aplicações. Rio de Janeiro: 2AB. 2009.

17. MORAES, Anamaria de, SANTA ROSA, José Guilherme. Design participativo: técnicas para inclusão de usuários no processo de ergodesign de interfaces. Rio de Janeiro: Rio Book's, 2012.

18. NEGRÃO, Celso; CAMARGO, Eleida. Design de embalagem: do marketing à produção. São Paulo: Novatec Editora, 2008.

19. PEDELHES, Gabriela Juppa. Embalagem: funções e valores na logística. Florianópolis: GELOG/UFSC, 2005.

20. PENHA, Anderson et al. $8 \mathrm{~min}$ of collaborative design. 2010. Disponível em: $\quad$ https://andrercoutinho.files.wordpress.com/2010/06/8-min-ofcollaborative-design.pdf. Acesso em: 23 abr. 2020

21. RODRÍGUEZ, Gerardo. Manual de diseño industrial: curso básico. [S. I.]: G. Gili, 1986.

22. RUDIO, Franz Victor. Introdução ao projeto de pesquisa científica. 23. ed. Petrópolis: Vozes, 1997.

23. TILLEY, Alvin R.; HENRY DREYFUSS ASSOCIATES. As medidas do homem e da mulher: fatores humanos em design. Porto Alegre: Bookman, 2005. 